\title{
Global protein profiling of porcine cumulus cells in response to native oocyte secreted factors in vitro
}

\author{
F. Paradis, H. Moore, S. Novak, M.K. Dyck, W.T. Dixon \\ and G.R. Foxcroft
}

Swine Reproduction-Development Program, Dept. AFNS, University of Alberta, Edmonton, AB, T6C 2P5, Canada

Until recently, the traditional view was that the oocyte played a passive role in folliculogenesis, relying on paracrine signalling from surrounding somatic cells to acquire developmental competence. However, recent evidence suggests that the oocyte plays an active role in the process of folliculogenesis by secreting soluble factors that act on the neighboring somatic cells. Studies using rodent and ruminant animal models have shown the importance of oocytesecreted factors for cell proliferation and differentiation, steroidogenesis, metabolism, and, in certain species, in determining ovulation rate. However, little information is available in the pig species. Therefore, the objective of the current study was to determine the effects of native oocyte-secreted factors on cumulus cell protein expression in the pig follicle.

Three groups of four sows were euthanized on day $19 \pm 1$ after the $1^{\text {st }}$ post-weaning oestrus at a time point when the pre-ovulatory follicle population was established and the oocytes should have acquired full developmental competence. Follicle size and follicular fluid oestradiol concentration were used to confirm that oocytes were derived from highly oestrogenic follicles that had not been exposed to the preovulatory-LH surge. Cumulus-oocyte complexes ( $\mathrm{n}$ $19 \pm 2$ per sow) were aspirated from these large pre-ovulatory follicles, washed three times in PVA TL-HEPES, and denuded (DO) from their cumulus cells. Concomitantly, gilt ovaries were obtained from a local slaughterhouse and oocytectomized cumulus complexes (OOX) were prepared by microsurgically removing the oocytes from their surrounding cumulus cells. Groups of 16 OOX were then cultured for $22 \mathrm{~h}$ with or without the DOs obtained from individual sows ( $n=4$ groups of OOX per treatment per replicate) in $36 \mu$ droplets of modified M199 medium under mineral oil at $38.5^{\circ} \mathrm{C}$ in a humidified atmosphere of $5 \% \mathrm{CO}_{2}$. For each of the three replicate cultures, 3 groups of $16 \mathrm{OOX}$ incubated with or without DOs were pooled and subjected to 2-dimensional gel electrophoresis on 7cm IPGstrips pH 3-10 in the first dimension and $10 \%$ SDS-PAGE slab gels in the second dimension. The SYPRO Ruby (BioRad, CA) stained gels were imaged on a Typhoon Trio (GE Healthcare). Due to incomplete isoelectric focusing for one replicate, only the gel images from the remaining two replicates were finally analysed using Progenesis SameSpots software (Nonlinear Dynamics, UK). Individual spot intensities were normalized with the total spot volume from the gel of origin. A preparative gel containing the protein from the cumulus cells of 500 cumulus-oocyte complexes was also run under the same conditions but using an $18 \mathrm{~cm}$ IPGstrip pH 3-10. The preparative gel was matched with the analytical gels and protein spots of interest were manually excised and sent to a mass spectrometry facility for identification by LCMS/MS (Centre Genomique du Quebec, Sainte-Foy, Canada). To confirm proper matching between the analytical and preparative gel, 6 abundant protein spots of interest from the analytical gel were also sent for identification.

More than 600 spots were matched across the 4 gels of which 14 proteins were found to be differentially expressed when the OOX were incubated alone or with DOs. Interestingly, 9 of 
the 14 differentially expressed proteins were up-regulated (1.2- to 2.7-fold change) in the absence of oocytes. Conversely, 5 of the 14 differentially expressed proteins were down-regulated in the OOX incubated without DOs; however, the magnitude of the down-regulation was lower, with only a 1.1 - to 1.2 -fold change, representing a $10-20 \%$ decrease in protein abundance.

These results suggest that removing the oocyte from the cumulus-oocyte complex leads to specific, and predominantly stimulatory, effects on cumulus cell protein expression. Amongst the 13 proteins that were identified, 5 up-regulated proteins and 1 protein that was down-regulated

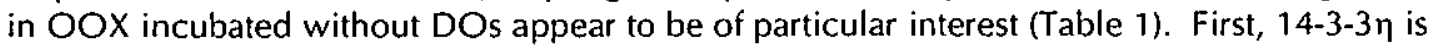
a member of the 14-3-3 protein family that modulates the interactions between proteins. The 14-3-3 proteins are involved in signal transduction and interact with various receptors including the glucocorticoid receptor, androgen receptor, insulin and IGF-1 receptor, as well as FSHR. In addition, they are also involved in the regulation of cell cycle progression, apoptosis and metabolism. NPM1 is a nuclear chaperone capable of acting as a transcription coactivator and modulates cell proliferation and apoptosis. P4HB is a key enzyme in collagen biosynthesis that appears to be essential in the peri-ovulatory period after the LH surge. Collagen synthesis is considered to be essential for repairing the ruptured follicle after ovulation but may also be necessary for the follicle-luteal transition that occurs at ovulation. TCTP is involved in modulating apoptosis but also alters cell morphology and positively regulates cell proliferation. The Rho GDP-dissociation inhibitor 1 prevents the activity of Rho GTPase which is involved in the organization of the actin cytoskeleton and in cell proliferation. Finally, PGAM1 is a glycolytic enzyme that catalyzes the interconversion of 2- and 3-phosphoglycerate. Changes in the activity of this enzyme modify the quantity of glucose 6-phosphate available for the pentose-phosphate pathway which ultimately modulates the amount of NADPH available for downstream biosynthetic pathways such as steroid biosynthesis. Altogether, these observations suggest that the porcine oocyte potentially modulates cumulus cells with regards to metabolism, apoptosis, proliferation and steroidogenesis. These findings are, therefore, consistent with the concept that oocytes secrete soluble factors that act on the cumulus cells to modulate their functions and phenotype.

Table 1: Identity of 6 proteins whose expression was differentially regulated when oocytectomized cumulus complexes $(O O X)$ were cultured with or without the denuded oocytes (DOs) harvested from large, oestrogenic porcine follicles that had not been exposed to the pre-ovulatory LH surge in vivo.

\begin{tabular}{|c|c|c|c|c|c|c|}
\hline $\begin{array}{c}\text { Spot } \\
\#\end{array}$ & $\begin{array}{c}\downarrow \uparrow \text { in OOX } \\
w / o \text { DOs } \\
\text { (fold change) }\end{array}$ & Protein name (symbol) & $\begin{array}{c}\text { Accession } \\
\#\end{array}$ & $\begin{array}{l}\text { Theoretical } \\
\text { MW (kDa)/pl }\end{array}$ & $\begin{array}{c}\text { \# of } \\
\text { unique } \\
\text { peptides }\end{array}$ & $\begin{array}{c}\text { Protein } \\
\text { coverage } \\
(\%)\end{array}$ \\
\hline 2 & $\uparrow(2.7)$ & $\begin{array}{l}\text { 14-3-3 protein eta } \\
\text { (YWHAH) }\end{array}$ & UPIO000EB0531 & $28 / 4.8$ & 21 & 60 \\
\hline 3 & $\uparrow(2.1)$ & Nucleophosmin (NPM1) & P06748 & $33 / 4.6$ & 10 & 39 \\
\hline 4 & $\uparrow(1.8)$ & $\begin{array}{l}\text { Prolyl 4-hydroxylase, beta } \\
\text { subunit }(\mathrm{P} 4 \mathrm{HB})\end{array}$ & P05307 & $57 / 4.7$ & 15 & 32 \\
\hline 5 & $\uparrow(1.7)$ & $\begin{array}{l}\text { Translationally-controlled } \\
\text { tumor protein (TCTP) }\end{array}$ & P13693 & $20 / 4.8$ & 6 & 31 \\
\hline 20 & $\uparrow(1.2)$ & $\begin{array}{l}\text { Rho GDP-dissociation } \\
\text { inhibitor } 1 \text { (ARHGDIA) }\end{array}$ & P19803 & $23 / 5.0$ & 8 & 43 \\
\hline 23 & $\downarrow(1.15)$ & $\begin{array}{l}\text { Phosphoglycerate mutase } 1 \\
\text { (PGAM1) }\end{array}$ & P18669 & $29 / 6.7$ & 20 & 78 \\
\hline
\end{tabular}

